\title{
Determinación geomorfológica de Choccoconniri - Puno
}

\section{Geomorphological determination of Choccoconniri - Puno}

\author{
Poma Porras Orlando Alan, Roman Medrano Maria Alejandra ${ }^{a}$, Zegarra López Zabdi \\ Magdalena $^{\mathrm{a}}$, Madrigal Perez Glen Bryan ${ }^{\mathrm{b}}$ \\ ${ }^{a}$ EP. Ingeniería Ambiental, Facultad de Ingeniería y Arquitectura, Universidad Peruana Unión, , Centro de Recursos en Geociencia - \\ Lima \\ ${ }^{b}$ EP. Ingeniería Ambiental, Facultad de Ingeniería y Arquitectura Universidad Peruana Unión, Centro de Recursos en Geociencia - \\ Sede Juliaca
}

\section{Resumen}

El objetivo esta investigación es determinar la geomorfología de Choccocconiri para mostrar relaciones existentes entre los procesos de cambio de esta, además la influencia climática en la modificación del relieve. La metodología empleada fue la observación panorámica con el dron Phanton 4 pro que hizo tomas con una altura de 50 metros y mediante imágenes satelitales, también la observación compuesta que consistió en recorridos por sectores para registrar de cerca características del territorio. La geomorfología registrada en Choccocconiri es consecuencia de los grandes eventos formadores de relieve ocurridos en el departamento de Puno, asociados a eventos tectónicos, transporte y depósito de material litológico así mismo a las modificaciones biológicas y climáticas, se observó gran variedad de geoformas y que son parte de diferentes geopaisajes, que están influenciados por la erosión eólica, acción fluvial y procesos de disolución química. En chocco el perfil edáfico es poco profundo con tres notables capas el primero de origen glaciar aluvial y en Cconiri es origen eólico fluvial y horizonte cálcico. La percepción de la población a través de bioindicadores como aves emigrantes y disminución del caudal de agua es que se están dando cambios notables en su comunidad. En conclusión, se encontró una geomorfología atractiva paisajísticamente, además, Estos procesos son todavía activos en rocas blandas, en la que los escarpes actuales están bien desarrollados. geoformas y piedras laja que son recursos geológicos de gran valor para la comunidad que permitirán, con un buen manejo, el desarrollo de la misma a través del ecoturismo.

Palabras clave: geomorfología; relieve; geopaisaje; Choccocconiri.

\begin{abstract}
Abstrac
The objective of this research is to determine the geomorphology of Choccocconiri to show existing relationships between the processes of change of this, in addition the climatic influence in the modification of the relief. The methodology used was the panoramic observation with the dron Phanton 4 pro that made shots with a height of 50 meters and through satellite images, also the composite observation that consisted of tours by sectors to register near characteristics of the territory. The geomorphology recorded in Choccocconiri is a consequence of the great formative events that occurred in the department of Puno, associated to tectonic events, transport and deposit of lithological material, as well as to biological and climatic changes, a great variety of geoforms were observed part of different geopajesajes, that are influenced by the wind erosion, fluvial action and processes of chemical dissolution. These processes are still active in soft rocks, in which the current escarpments are well developed. In chocco the edaphic profile is shallow with three notable layers the first of alluvial glacial origin and in Cconiri it is fluvial wind origin and calcic horizon. The perception of the population through bioindicators as migrating birds and decreasing water flow is that they are giving remarkable changes in their community. In
\end{abstract}


conclusion, an attractive geomorphology was found in the landscape, in addition, geoforms and slab stones that are geological resources of great value for the community that will allow, with a good management, the development of the same through ecotourism.

Keywords: geomorphology; relief; geopasaje; Choccocconiri.

\section{Introducción}

En la corteza terrestre se encuentran geoformas las cuales atraviesan diversos cambios algunos de ellos de manera natural como el resultado del paso del tiempo, la gravedad, precipitación, entre otros y lo cambios producidos de manera antrópica que es una variable resaltante para el estudio de los cambios en la corteza (Flores, 2003) donde todo lo que ella compone se ve afectado de manera drástica cuando existen cambios, los espacios naturales en el mundo tienen ciclos únicos, y también una capacidad de autorregulación especial en cada caso sin embargo Arribas et al., (2012) afirma que el cambio climático al que nos enfrentamos en la actualidad constituye una de las principales amenazas para el futuro de la biodiversidad. Las condiciones climáticas y edáficas de las montañas las hacen más susceptibles a la recuperación de los procesos regenerativos naturales, debido a las temperaturas más frías así también como el mayor potencial de erosión debido a las mayores pendientes, y los suelos en general menos fértiles son algunos elementos que hacen peligrar el medio ambiente natural de las montañas y la fertilidad de sus suelos, debido al efecto que tiene la acción del hombre en la disminución del área y la fragmentación de los hábitats que ocupan las especies (Garreaud, 1998).

Frente a la problemática del mal uso de las áreas naturales y la pérdida de la biota en las zonas de montaña, la educación ambiental juega un papel clave en la concientización para la conservación de espacios naturales con potencial paisajístico ya que puede llegar a ser una fuente de ingresos por medio del ecoturismo.

\section{Factores de modificación del relieve}

La geomorfología como ciencia se encarga del estudio de la formación, procesos y formas de relieve, que implica además el estudio de geológico, el uso del suelo y la hidrología in situ. Es fundamental para el mapeo y caracterización de los suelos, los riesgos y amenazas, el mapeo de la erosión, modelamiento de glaciares, climatología y en forma indirecta, en estudios de ecología, vegetación, agricultura y uso de la tierra (Laubacher, 1978). El objeto de estudio de la geomorfología son las geoformas las cuales tienen características morfográficas, morfométricas, morfogenéticas y morfocronológicas. Es decir, una geoforma es también un término usado para referirse a todos los tipos de formas del relieve (niveles 
taxonómicos), independientemente de su origen, dimensión y nivel de abstracción (Palacios, 2002). El relieve terrestre va evolucionando mediante una serie de procesos destructivos y constructivos que se ven afectados por la gravedad (las zonas elevadas caigan y colmatan las zonas deprimidas) estos procesos son desencadenados por procesos geomorfológicos que son:

- Factores geográficos: entre los que se consideran los factores abióticos de origen exógeno, tales como el relieve, el suelo, el clima (presión, temperatura y vientos) y los cuerpos de agua (agua superficial, con la acción de la escorrentía, la acción fluvial y marina, o los hielos en el modelado glacial).

- Factores bióticos: El efecto de los factores bióticos sobre el relieve suele oponerse a los procesos del modelado, especialmente considerando la vegetación, sin embargo, existen pocos animales que colaboran con el proceso erosivo

- Factores geológicos: tales como la tectónica, el diastrofismo, la orogénesis y el vulcanismo, son procesos constructivos y de origen endógeno que se oponen al modelado e interrumpen el ciclo geográfico.

- Factores antrópicos: La acción del hombre sobre el relieve es muy variable, dependiendo de la actividad que se realice, en este sentido es muy difícil generalizar, pudiendo incidir a favor o en contra de los procesos erosivos.

Existen por lo tanto factores que desatan los procesos geomorfológicos que pueden ser endógenos o exógenos encargados de dar forma de relieve en la superficie del planeta; éstos están diferenciados en tres campos: Geomorfología climática, geomorfología estructural y geomorfología dinámica (Petersen, 1980).

\section{Geomorfología Climática}

Estudio causa efecto del comportamiento del clima y la resultante modificación de los relieves, que como resultado en el modelado se requieren datos de la influencia del clima de la zona de estudio la cual es manifestada por el tipo de erosión en el relieve ya sea por erosión diferencial de la estructura geológica y litológica así como de otras variables. Según la petrografía de las rocas y el clima, ellas tienen distintos comportamientos, se muestra primero cuando el clima puede actuar de manera directa sobre la superficie terrestre o indirectamente cuando existe cubierta vegetal. De este modo, el efecto del clima depende de la cubierta vegetal y de los suelos (Rivera, 2011).De acuerdo con lo mencionado, se reconocen sistemas morfogenéticos en que dominan procesos geomorfológicos con acciones 
mecánicas y, sistemas morfogenéticos en que dominan los procesos físico-químicos y bioquímicos (Rose, 1979).

En el Cuaternario, había variaciones climáticas importantes también ya que ahora se pueden ver de manera significativa sus efectos en la geomorfología del relieve estas son caracterizadas por la alternancia de los períodos glaciales e interglaciales. Este hecho se muestra de manera sucesiva contribuyendo en la morfogénesis que es discontinua en el tiempo y en el espacio. El período Cuaternario es de gran inestabilidad climática incluso se muestran modelados actuales en la superficie terrestre que son heredados de paleoclimas (Huang, 1991).

Es así como el hecho de que la herencia del efecto climático en el relieve hace posible encontrar formas que no corresponden al conjunto coherente de las otras formas dentro de las cuales se localizan y que están sometidas a la acción de procesos que no tienen relación directa con las características de su modelado (Hartman, 1987). Estas formas heredadas son el testimonio de épocas anteriores en que fueron elaboradas bajo otras condiciones climáticas diferentes de las actuales. Algunos ejemplos de geoformas que se originaron en períodos glaciales y que hoy se encuentran en áreas donde no hay glaciares son los lagos de represamiento morrénico, los circos y los amplios valles glaciales. De los períodos interglaciales se reconocen en algunas áreas ciertos depósitos y formaciones superficiales tales como playas de niveles marinos transgresivos, alteraciones rojizas y costras ferruginosas. La diversidad de climas representa distintas de velocidades en la evolución del ciclo ej.

- Climas áridos con ritmo evolutivo más lentos

- Climas muy húmedos con ritmos evolutivos más alto

Del mismo modo también el clima representa el tipo de modelado predominante ya sea glacial, eólico, fluvial, etc. con el fin de agrupar las zonas climáticas ya sean: templados, intertropicales, áridos, subáridos, glaciares o árticos y periglaciares o subárticos. Así mismo este estudio permite caracterizar los rasgos geomorfológicos de los diferentes tipos de rocas ya sean de relieves volcánicos, relieves graníticos, relieves cársticos, etc.) (Chavez, Salas, \& Cuadros, 1996)

\section{Geomorfología Estructural}

Estudio de relaciones entre procesos endógenos y formación geológica de estructuras en el área, trata los fundamentos litológicos y tectónicos los que definen la forma y el tipo de relieve en la superficie terrestre eso incluye los contactos de éste como es el caso de la hidrografía a diferentes escalas. Existen dos movimientos básicos los verticales o 
epirogénicos de amplio radio y muy lentos, que tratan de recuperar el equilibrio isostático; y los movimientos horizontales u orogénicos, responsables de los relieves plegados y fracturados. En la actualidad el paradigma que explica el relieve de la Tierra es la tectónica de placas (David, 1992).

\section{- Epirogénesis}

Es referido al movimiento del tipo vertical en la corteza terrestre a escala continental las que afectan a grandes áreas interiores de las placas continentales: plataformas y cratones. Son movimientos de ascenso o descenso muy lentos sostenidos (no repentinos) que pueden tener como consecuencia el basculamiento de una estructura como la ocurrida en la península ibérica durante el terciario que tuvo como consecuencia el drenaje de los lagos interiores hacia en atlántico. También pueden tener como resultado grandes abombamientoses decir cuando se generan estructuras aclinales (no plegadas). Si el abombamiento es ascendente, o positiva, se llama anteclise; y si el abombamiento es descendente, o negativa, se llama sineclise. En las anteclise predominan las rocas de origen plutónico en contraste en las Sineclise funciona como cuencas de acumulación por lo que predominan las rocas sedimentarias. Figura1.

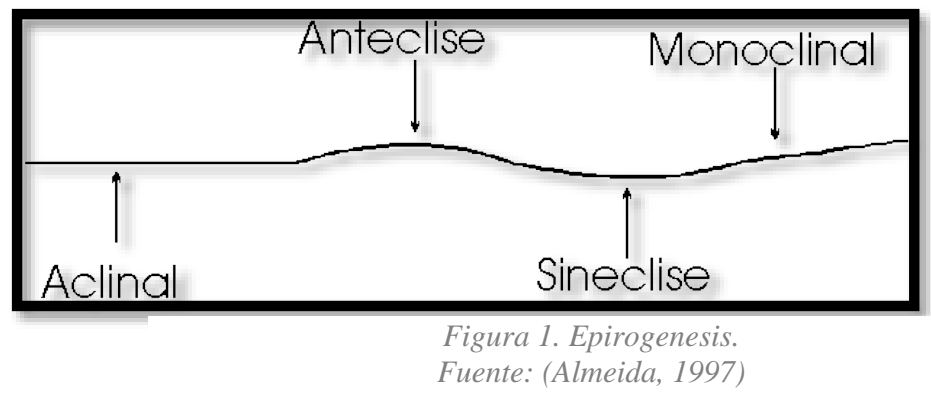

- Tectogénesis u Orogénesis

Los movimientos orogénicos son los movimientos horizontales de la corteza terrestre, teniendo en cuenta que la Tierra es una esfera. Afecta a regiones relativamente pequeñas, aunque de manera generalizada; las grandes orogenias han afectado a todo el globo, pero se expresan puntualmente y en forma de crisis. Son movimientos relativamente rápidos.

\section{Geomorfología Dinámica}

Estudio analítico del cambio de relieve (diferencia entre elevaciones) y elevación (altura de un punto sobre el nivel del mar), por agentes geomorfológicos, agentes que favorecen la meteorización, erosión y transporte de material, todo ello en función a su 
aspecto, origen y evolución. Son dos procesos principales que originan las formas de relieve terrestre (Acosta, Rivera, Valencia, Chirif, \& Huanacuni, 2010).

El primero el cual es el formador del relieve y está relacionado con los procesos internos de la corteza el cual incluye las tectónicas de las placas, llamados procesos constructivos este proceso es llamado endógeno. El segundo se refiere a los modeladores de relieve que incluye los procesos del ciclo sedimentario lo que incluye procesos destructivos (destruyen el paisaje y rocas que los conforman, modelando las formas del relieve) y acumulativos (acumulan sedimentos modelando la forma del relieve), este es debido a la acción erosiva del agua, hielo, viento y gravedad.

\section{- Procesos endógenos}

Se deben a la tectónica de las placas y da lugar a dos tipos de relieve: Forma de relieve en el continente y forma de relieve en la corteza oceánica, el proceso de formación más importante en esta investigación es el de las formas de relieve en el continente en cual se puede dividir en tres regiones: cadenas montañosas, plataformas y escudos como se observa en la Figura 2 (Betejtin, 1975).

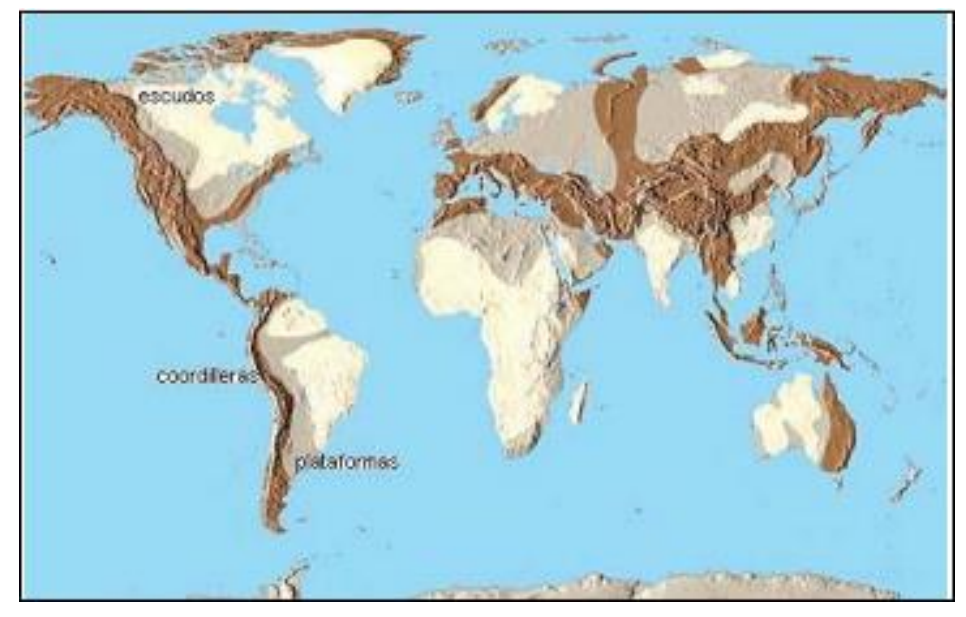

Figura 2. Formas de relieve en el continente. Fuente: Caballero M. (1992).

- Cadenas montañosas. - Son zonas con grandes diferencias de relieve que se forman debido a los procesos de choques de placas, justificando su ubicación en los límites de convergencia; En estas zonas la corteza se engruesa y las fuerzas de comprensión forman montañas las cuales son denominadas tectónicamente antiguas (límite con placas actuales) o suturas (límite con placas antiguas). En américa las principales son: 
Cordillera de los andes en el margen del pacífico, aún activas y los Apalaches en la margen oriental corresponde a sutura.

- Plataformas.- Denominadas formas tectónicamente estables ya no están cerca del límite de placas, estas presentan pocas diferencias de relieve, son de poco espesor y poca deformación.

- Escudos.- Constituidas por rocas muy antiguas, las más antiguas de la tierra (precámbricas) además cubiertas y rodeadas por estratos más jóvenes; están ubicadas en regiones geológicamente estables.

\section{Procesos Exógenos}

Los procesos que forman el relieve son originados por la acción de diversos procesos que ocurren en la tierra. Estos procesos están incluidos en el ciclo de las rocas que en gran medida están ligadas por el ciclo del agua y por lo tanto sometidas al clima dominante en la región. Se presentan a continuación los agentes modeladores del paisaje, cada uno de ellos actúa en determinados ambientes y dan lugar a procesos característicos. tabla1. Cabe mencionar además que los procesos exógenos dan lugar a dos tipos de relieves: Formas erosivas (dominio de remoción de material) y formas acumulativas (acumulación de material) (Castillo, Inche, \& Rivera, 2011)

Tabla 1. Procesos exógenos que dan lugar a dos tipos de relieve: Erosivas y Acumulativas.

\begin{tabular}{|c|c|c|c|}
\hline Agentes & Procesos/ Ambiente & $\begin{array}{l}\text { Formas de relieve } \\
\text { erosivas }\end{array}$ & $\begin{array}{l}\text { Formas de relieve } \\
\text { Acumulativas }\end{array}$ \\
\hline Gravedad & $\begin{array}{l}\text { deslizamiento de } \\
\text { masas, inestabilidad } \\
\text { laderas }\end{array}$ & $\begin{array}{l}\text { "cicatrices de } \\
\text { deslizamientos" }\end{array}$ & $\begin{array}{l}\text { Caídas/flujo de detritos, } \\
\text { avalanchas, deslizamientos }\end{array}$ \\
\hline Hielo & Glaciares & $\begin{array}{l}\text { circos, horns, aristas, } \\
\text { valles en } U\end{array}$ & Morrenas, drumlins, eskers \\
\hline Agua & $\begin{array}{l}\text { Fluviales (fluvio- } \\
\text { aluvial) }\end{array}$ & $\begin{array}{l}\text { circos y terrazas de } \\
\text { erosión, valles en } V\end{array}$ & $\begin{array}{l}\text { Abanicos aluviales, terrazas } \\
\text { de acumulación, bancos de } \\
\text { ríos }\end{array}$ \\
\hline Viento & Eólicos (desértico) & $\begin{array}{l}\text { Erosión diferencial, } \\
\text { yardangs, facetas de } \\
\text { erosión, pavimento del } \\
\text { desierto }\end{array}$ & Dunas, barjanes, loess \\
\hline $\begin{array}{l}\text { oleaje y } \\
\text { corrientes } \\
\text { marinas }\end{array}$ & Costero/mixto & $\begin{array}{l}\text { Terrazas de erosión y } \\
\text { acantilados }\end{array}$ & $\begin{array}{l}\text { Terrazas de depósito, bancos } \\
\text { de } \\
\text { arena, tómbolos, deltas }\end{array}$ \\
\hline $\begin{array}{l}\text { oleaje } y \\
\text { corrientes } \\
\quad \text { marinas }\end{array}$ & $\begin{array}{l}\text { Plataforma y talud } \\
\text { continental }\end{array}$ & $\begin{array}{l}\text { Arrecifes, (atolones, } \\
\text { guyots) }\end{array}$ & Abanicos abisales \\
\hline
\end{tabular}


Modificado por Zegarra (2017)

Es necesario destacar además en el estudio de la geomorfología dinámica las etapas de evolución de un paisaje importantes para esta investigación. Las etapas de evolución de un paisaje según el ciclo de Davis son: relieve joven, maduro y viejo. Sólo valido para relieve en rocas sin deformar (Figura 3). Aunque este modelo no es aceptado por su concepción muy general es una buena base para entender el comportamiento y evolución del paisaje. Actualmente existe otro modelo denominado equilibrio dinámico, donde se presentan también los diferentes agentes comprometidos donde el paisaje es una adaptación dinámica

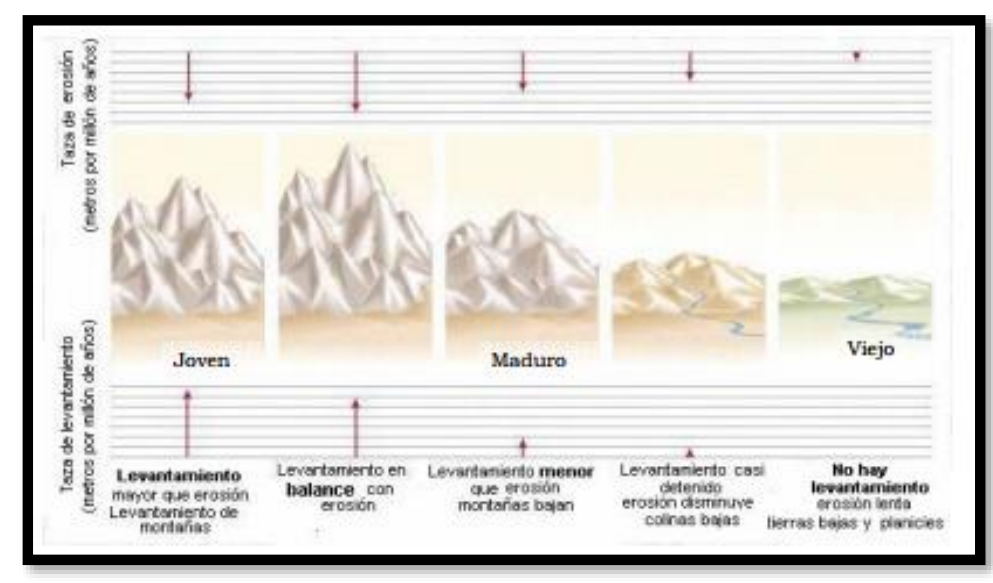

Figura 3. Etapas de evolución de un paisaje.

Fuente: (Chávez, 2011).

a la estructura de las rocas, clima, relieve local y altura manifestado a través del tiempo; Sin embargo terremotos y erupciones (procesos endógenos) y las precipitaciones o incendios (procesos endógenos) son también capaces de proporcionar significativas variaciones de relieve que actúan condicionando o evolucionando el paisaje (Chávez, 2011).

Las etapas de evolución de un paisaje tienen un desarrollo complejo de erosión y levantamiento. Un claro efecto es la relación de elevación-erosión, es decir mientras mayor 
sea la elevación mayor será la erosión y si la elevación es menor, menor también será la erosión (Figura 4).

\section{Otros controles de relieve}

Otros métodos de controlar el relieve son: composición y granulometría de la roca, y estructura geológica. Para el primero es decir para el tipo de roca, su estudio ya sea composición química o tamaño de los componentes pueden controlar el desarrollo de las laderas de pendientes suaves o fuertes (el tamaño de las partículas finas: menor pendiente; partículas gruesos: mayor pendiente). Figura 5. La estructura de las rocas pueden controlar la pendiente de las laderas, así también la formación de valles y patrón de drenaje. Es

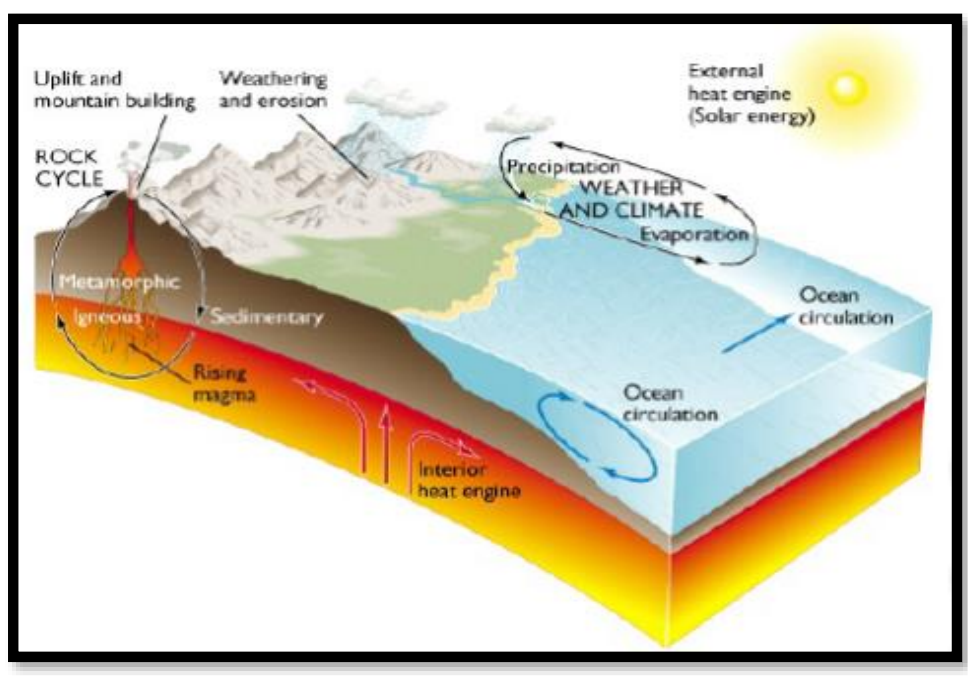

Figura 4. Ciclo de Davis 1989, modelo de evolución del paisaje. Fuente: (Chávez, 2011)

importante recalcar además que el drenaje (patrón de drenaje, densidad y profundidad de disección) controlan el tipo de roca y su estructura ya que si una roca es impermeable, tendrá gran densidad de arroyos (Ej. Arcillas), si la roca es permeable tendrá menos cantidad de arroyos (Ej. Arenisca), Figura8. (Goldschmidt, 1937)

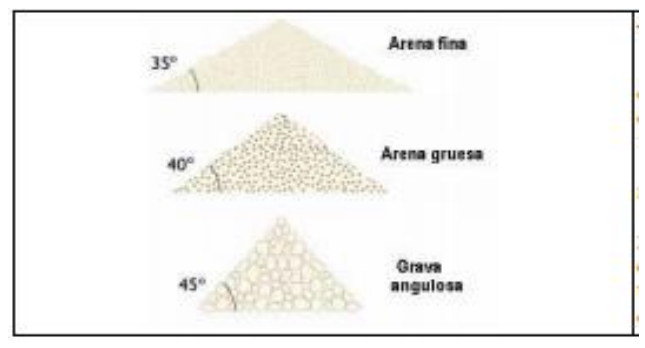

Figura 7. Pendiente de laderas. (Chávez, 2011) 


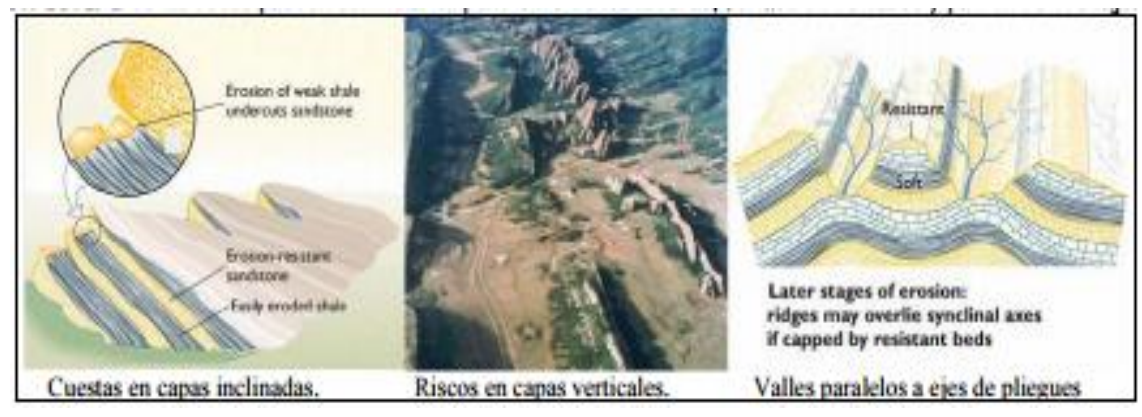

Figura 5. Estructuras de las rocas.

Fuente: (Betejtin, 1975)

La tierra (superficie, sentido; suelo; drenaje y formas minerales) que incluye procesos edáficos y climáticos, son estudiados por la geomorfología la cual se ocupa de la historia, la fisiografía es decir los procesos y la morfología de la geografía que se ocupa de la descripción. Todos los procesos dados para la formación del relieve se pueden interpretar en el mapa topográfico en términos de uso y forma de dicha superficie ligados de manera análoga con el clima de la zona (Palacios, Geológico detallado de la Región Sur Oeste del Perú., 2002).

Un claro indicador de cambios de clima es debido al tipo de vegetación desarrollada de acuerdo con la altura y ubicación, por lo tanto, la diversidad es una propiedad intrínseca del sistema viviente, donde la pérdida de las especies presentes significaría la pérdida del ecosistema. En la presente investigación se determinará la geomorfología de Choccocconiri en Juli-Puno (Sillitoe, 2010).

Puno está ubicado en el Collao, altiplanicie que desde la región del Cuzco se extiende casi 800 kilómetros hacia el sur, hasta el lago Poopó, en Bolivia, a una altura que oscila entre los 4200 y los 4600 metros sobre el nivel del mar. A manera de un colosal anfiteatro, este vasto escenario se halla flanqueado, hacia el noreste, por la cordillera Oriental, montaña de topografía muy escarpada, seccionada por valles profundos y encañonados que dan lugar, entre ellos, a alineamientos como la cordillera de Carabaya y que luce imponentes picos, como el Allin Ccápac (5850), el Ritipata (5350) o el Balansani (5354) y hacia el noroeste, por las cumbres nevadas de la Cordillera Occidental entre otras, el Cunurana (5420), el Lamparasi (5327), y la cadena volcánica de la que forman parte el Ubinas (5672), el Tutupaca (5815) y el Ticsani (408) (Tumialan, 2003).

El altiplano es actualmente una típica superficie de erosión. La parte sur del Collao alberga la depresión del Titicaca, de origen tectónico, que corre del noroeste al sureste por más de trescientos kilómetros, con un ancho de hasta cien kilómetros, y en el centro de la cual se encuentra el lago del mismo nombre. Aquí la uniformidad del terreno es, si cabe, mayor, pues ésta es una zona de extensas llanuras aluviales cubiertas de pastos y matorrales, como las de Lampa, Juliaca y Ayaviri. 
De los 72382 de kilómetros cuadrados del departamento de Puno, aproximadamente el 70\% está ocupado por la altiplanicie del Collao. El 30\% restante lo constituyen los terrenos cordilleranos y la zona de selva que limita con Madre de Dios.

\section{Descripción del área de estudio}

Juli ubicado a 3819 m.s.n.m., conocida como "La Pequeña Roma de América” cercana a la orilla (Sur Oeste) del Lago Titicaca, que está ubicada en las faldas de los cerros Pucara, San Bartolomé, Sapacollo y Q'aracollo Extensión: Tiene una extensión de 1,650 Km2. Su topografía presenta planicies, valles, cataratas, quebradas, llanuras. En Juli se encuentra el centro poblado de Choccocconiri, con temperatura max de $12.8^{\circ} \mathrm{C}$ y $\min .5 .9^{\circ} \mathrm{C}$. Choccocconiri, el área de estudio está compuesto por las zonas Chocco y Cconiri ambas zonas con elevaciones muy diferenciadas.

\section{MAPA DE UBICACIÓN DE CHOCCOCONIRI}

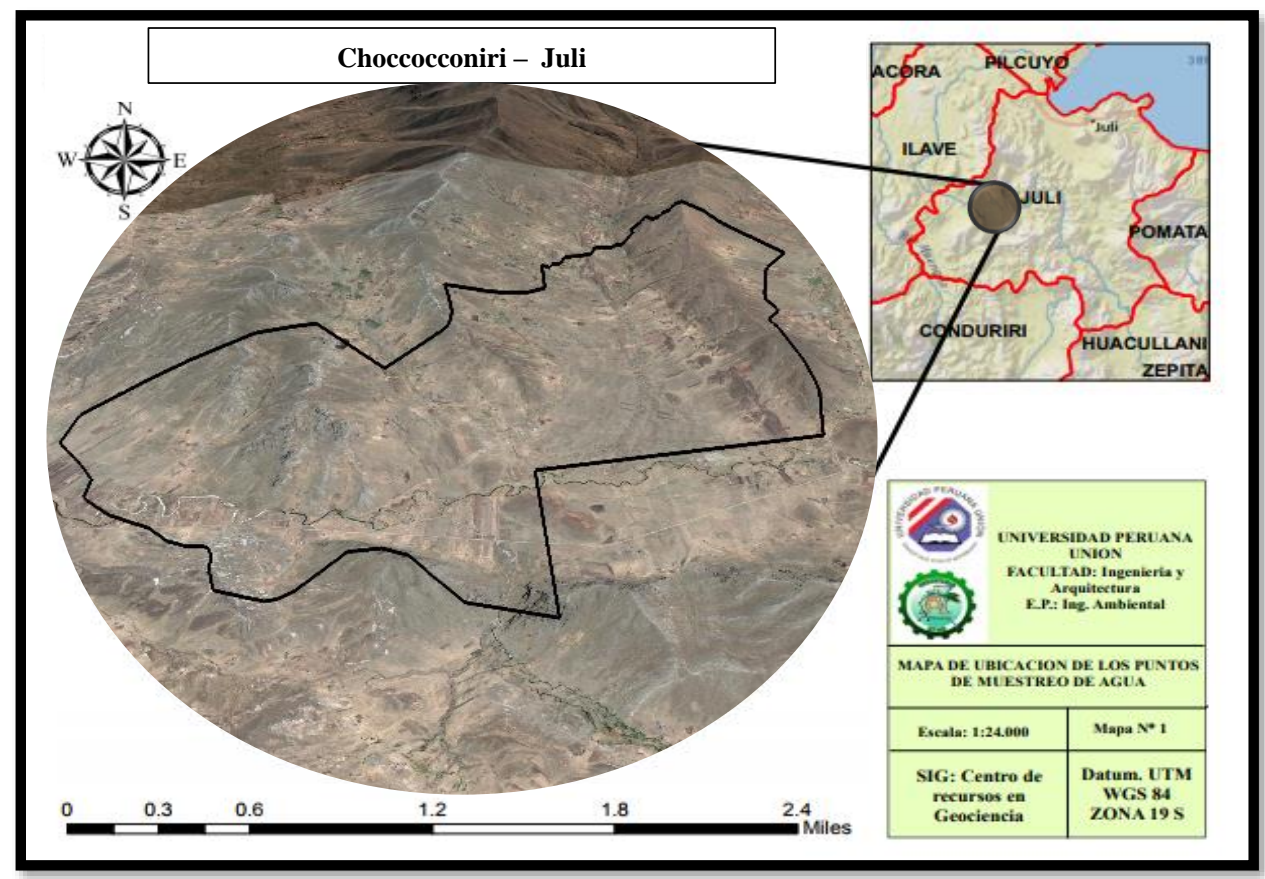




\section{Materiales y Métodos}

Los materiales utilizados en campo fueron la cámara fotográfica, el dron Phanton 4 pro, GPS Garmin Etrex 20, Laser trupulser, libreta de apuntes.

\subsection{Método de observación panorámica}

Este método permite gran cobertura y mejor entendimiento de las diferentes zonas que se va a recorrer, además permite capturar imágenes de lugares muchas veces inaccesibles, dan una mejor idea de la geomorfología y permiten entender sus formas con claridad ya que se aprecian ríos pendientes y otros factores que en recorridos no son fáciles de distinguir, la distribución más general es la que se aprecia en el siguiente gráfico.

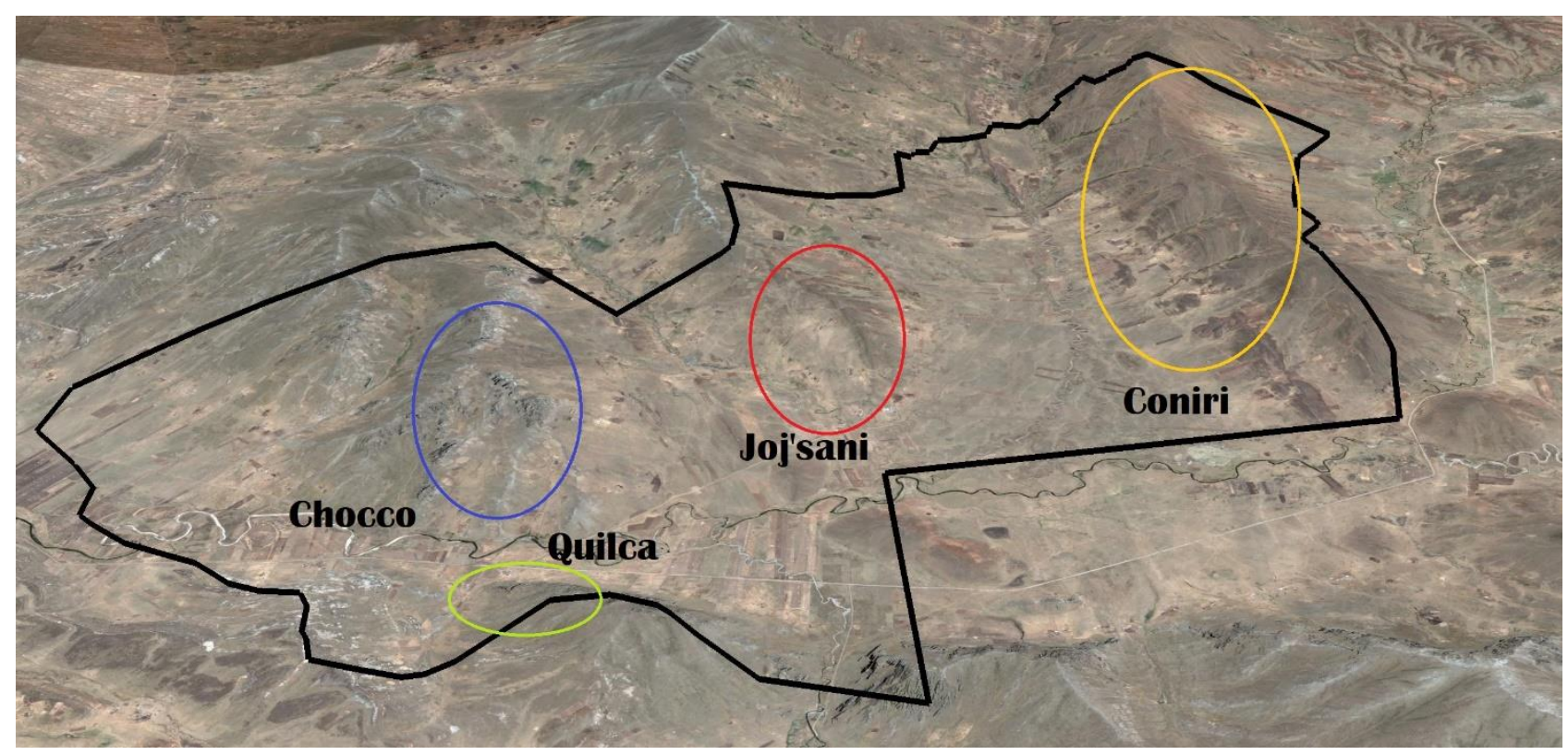

Figura 7. Sectores en el territorio de la comunidad de Choccocconiri Elaborado por: Roman M. (2017)

\subsection{Método de la observación compuestas}

La imágenes panorámicas, permiten amplia visibilidad pero sin la observacion cercana no se puede conocer otros componentes y procesos que se estan dando en las rocas, es por eso tan importante la observación compuesta que consiste en registrar datos insitu y luego todo el material en digital analizarlo y compararlo.

Se realizaron visitas de campo, a las zonas sur, sureste y noreste de la zona de estudio mediante recorridos vehiculares por la red vial secundaria existente en Juli- Puno. Se reconoció el área de trabajo, se tomó fotografías panorámicas de las áreas que componen Choccocconiri, se tomó los puntos de ubicación GPS del área, para tener el conocimiento de 
la geomorfología en ambas zonas. Durante esta etapa de fotografiaron estructuras, elevaciones, formas etc. mientras se obtenían datos geológicos característicos mediante la observación.

\section{Resultados}

\subsection{Geomorfología de Chocco}

En general, la geomorfología de Chocco está influenciada por la erosión eólica, acción fluvial y procesos de disolución. Los escarpes actuales están bien desarrollados. Su relieve es empinado es plana o ligeramente ondulada con una débil inclinación hacia el río Haruma. Pertenece al gran grupo Ustorthent, según el Soil Taxonomy (2014).

Estos suelos se han formado a partir de materiales de origen fluvioglaciar. Se encuentran distribuidos en superficie de relieve empinado en la parte occidental. El perfil del edáfico es profundo, de textura media a gruesa, de reacción ácido y drenaje bueno a moderado. Generalmente estos suelos presentan cierto contenido de gravas y guijarros como piedras en zonas de menor pendiente.

Estas áreas misceláneas identificada como glaciar. En Cchoco presentan áreas de perfil AC poco evolucionado, la fertilidad de la capa arable es media.

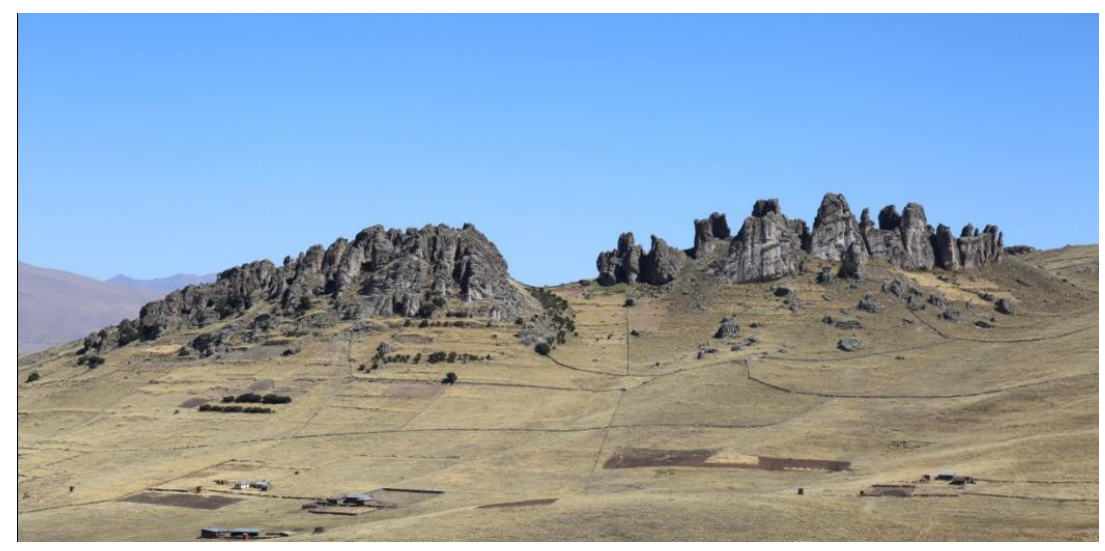

Figura 8. Geomorfología de Chocco. Fuente: Dron Phanton 4 Pro dirigido por Cunza (2017) 


\subsubsection{Erosión de rocas}

En los recorridos por la zona en todos los sectores se aprecian rocas en estado de erosión, la erosión por factores del clima y por agentes biológicos es muy apresurada dando lugar a atractivos geopaisajes, Esto en todos los sectores.

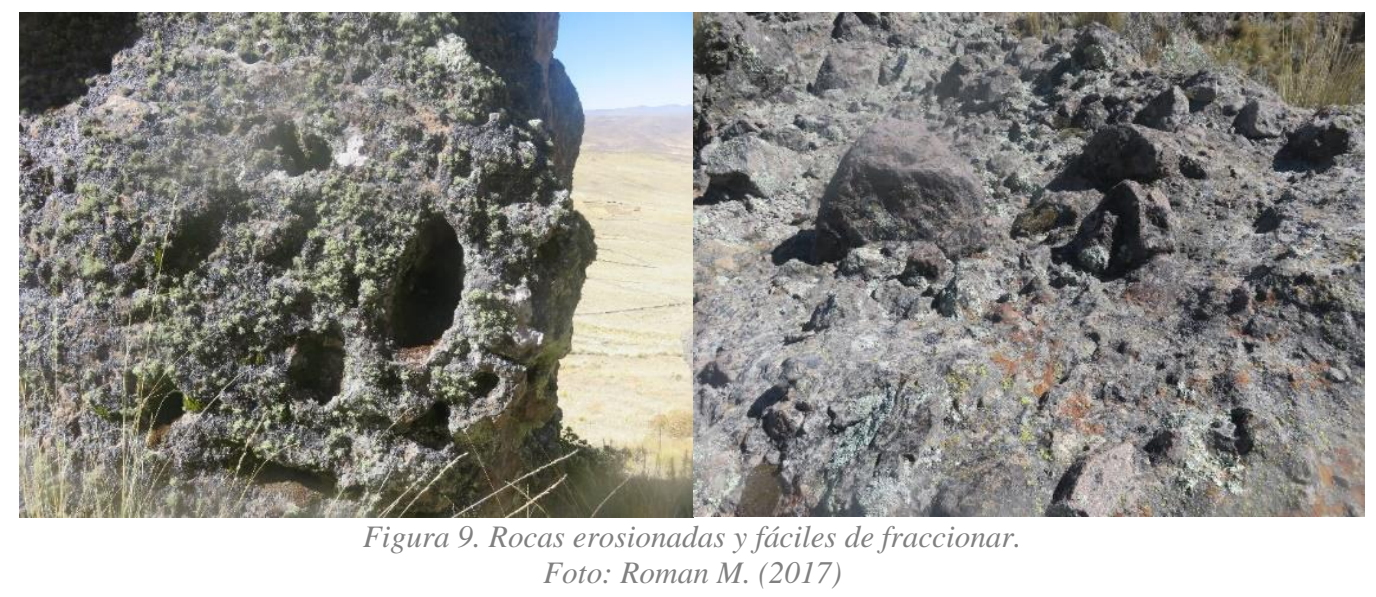

\subsubsection{Gran variedad de groformas}

En todo el recorrido por Isca Choco y Jacha Choco se observa una gran variedad de geoformas, tal fue que quedaron identificadas 16.

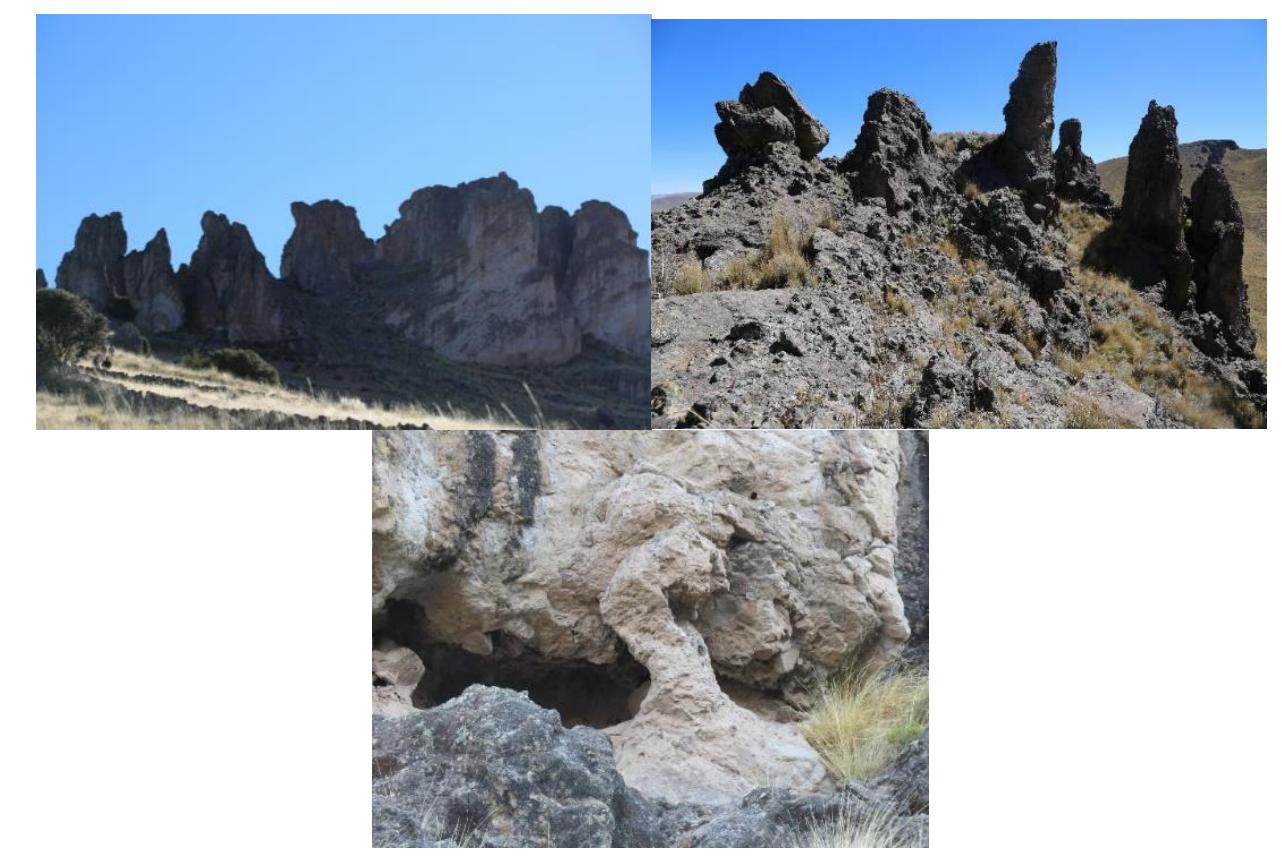




\subsection{Geomorfología de Quilca}

En este territorio se observe la mayor atraccion de formaciones rocosas elevadas con grandes alturas entre los 18 y 50 obtenidos con el lase trupulser.

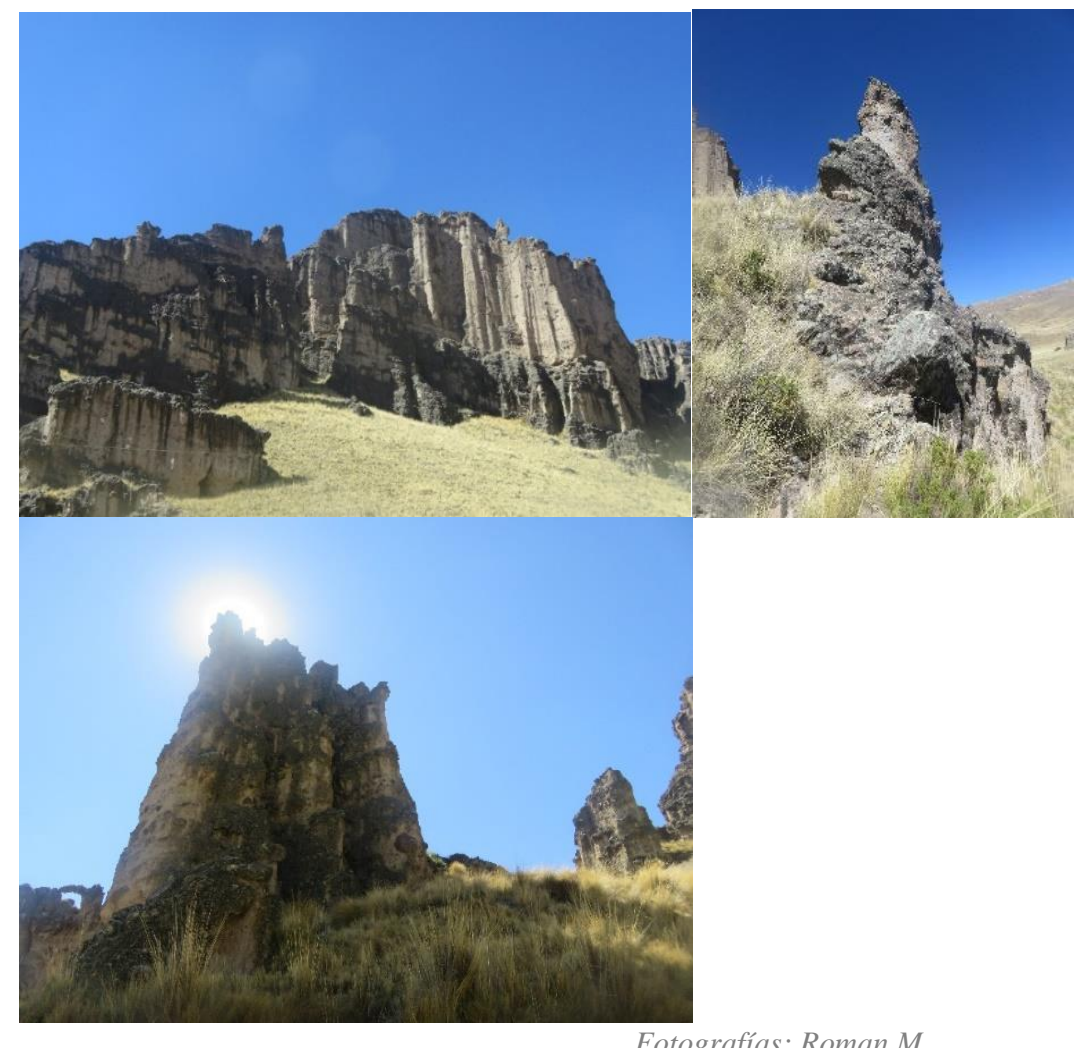

Figura 11. Grandes elevaciones con pendientes verticales

\subsection{Tomas panorámicas del Dron Phanton 4 pro}

Los imagenes captadas por el dron Phanton 4 pro fueron escenciales para el studio del relieve ya que con una visión clara a una Altura de aproximadamente 50 metro y a una altitud de $4141 \mathrm{msnm}$ se obtienen datos reales insitu que nos permieten fortalecer una valoración positive a todos estos paisajes.

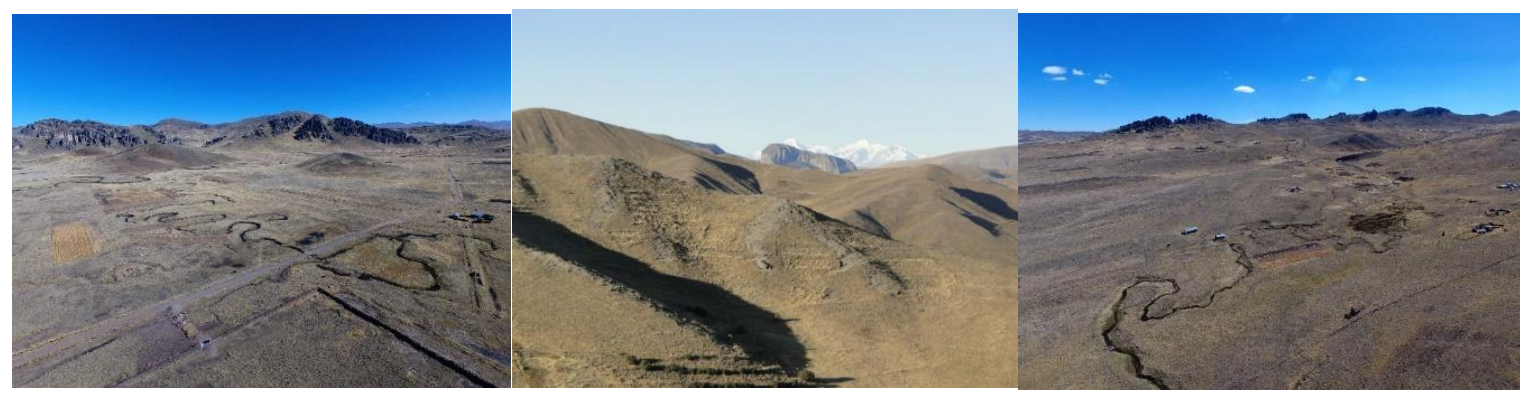

Figura 12. Vistas panoramicas con el dron Phanton 4 pro 


\subsection{Geomorfología de Cconiri}

En general, la geomorfología de Chocco está influenciada por la erosión eólica, acción fluvial y procesos de disolución. Estos procesos son todavía activos en rocas blandas, en la que los escarpes actuales están bien desarrollados. Su topografía es plana o ligeramente ondulada con una débil inclinación hacia el río Haruma. Pertenece al gran grupo Ustorthent, según el Soil Taxonomy 2014.

Está formado por el suelo de Cconiri. Estos suelos se formaron a partir de acumulados por acción de precipitaciones y gravedad, El perfil edáfico es profundo, de evolución variada de textura media a gruesa de reacción ácido y drenaje bueno a moderado. Generalmente estos suelos presentan cierto contenido de gravas y guijarros en su perfil, así como piedras. Se presenta en fase por pendiente empinada. Los suelos son de origen aluvial y se encuentran en las altiplanicies. Presenta un perfil ABC es decir poco evolucionado, identificándose un epipedón Ocric y un sub horizonte Cálcico, como horizonte de diagnóstico. Los suelos son moderadamente profundo; con limitaciones de factor suelo, erosión y clima, la textura media, la reacción es moderadamente alcalina, la saturación de bases está alrededor de 100\%, presentando carbonatos. La fertilidad de la capa arable es media.

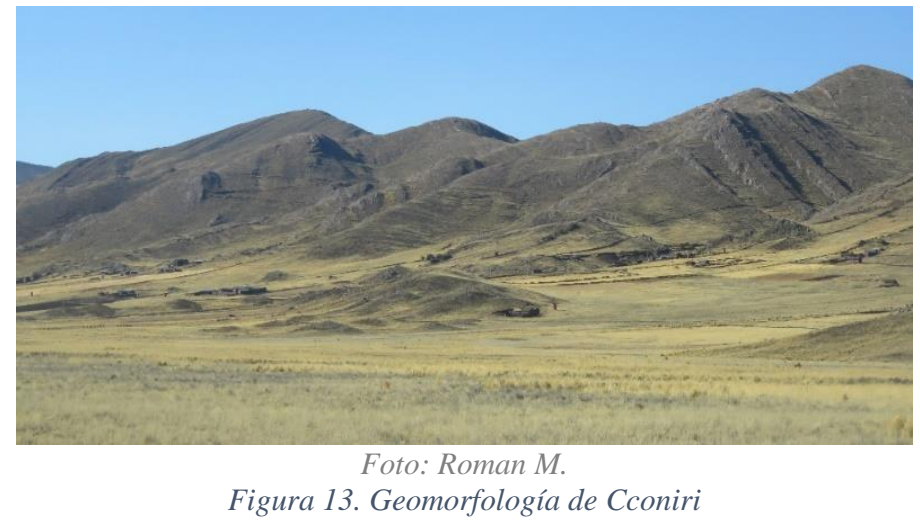

Las piedras laja de la zona de Conirir son un gran recurso geológico con el que cuenta la comunidad además las formas amplias de las arquetas en sus cerros resultan un atractivo, esto resulta importante material geológico, que deben aprovechar como recurso los comuneros. 


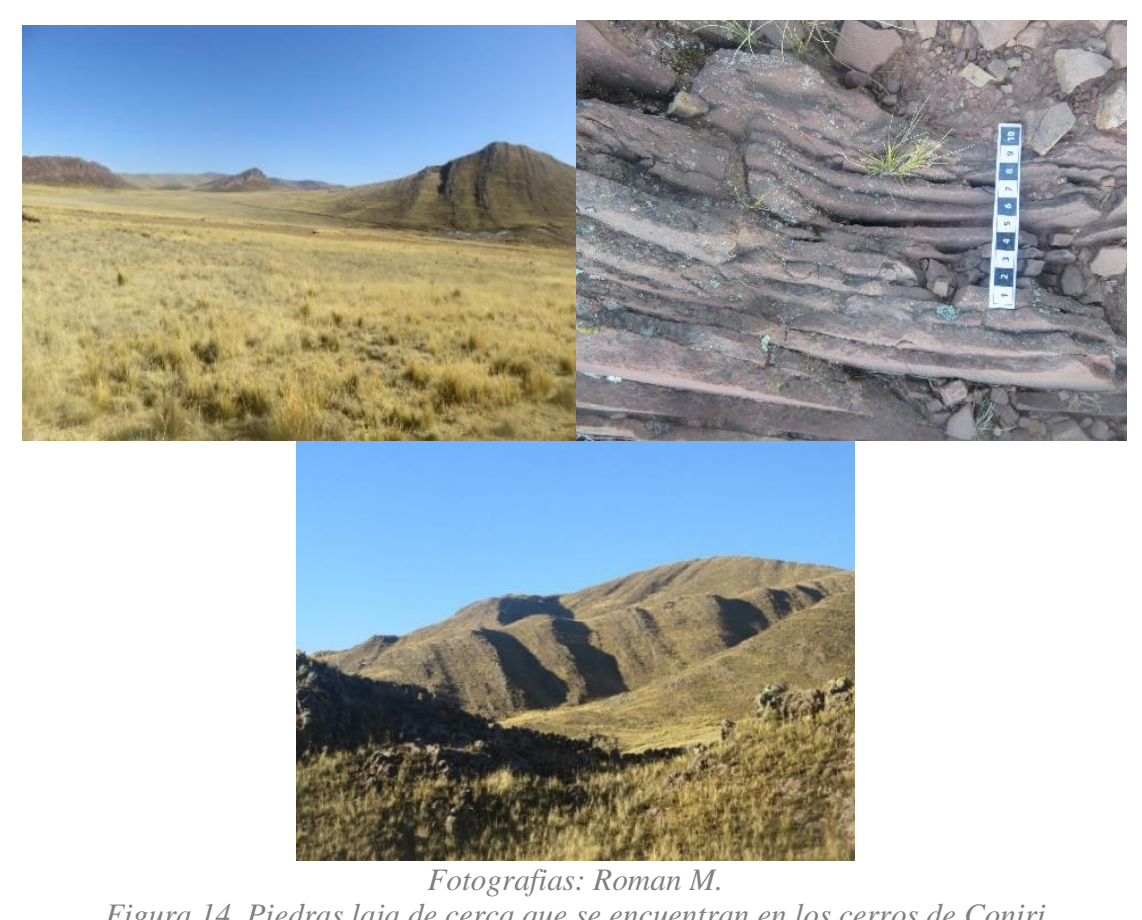

Figura 14. Piedras laja de cerca que se encuentran en los cerros de Coniri

\section{Conclusiones}

El clima es un factor importante para la determinación de muchos de los factores que son la base en el proceso de erosión, en diferentes tipos de roca o material. El efecto del cambio climático en los últimos años modifica y altera los espacios naturales, modifica el suelo, el relieve; además del cambio de temperaturas limita el crecimiento o desarrollo de vida de diversas especies y esto también modifica el paisaje, es importante recalcar el cuidado del mismo y su preservación consecuentemente al conocer la importancia de los espacios paisajísticos y los beneficios que otorga la comunidad con un buen manejo de los recursos geológicos podrán desarrollarse a través del ecoturismo.

\section{Recomendaciones}

Se recomienda identificar qué servicios ambientales y bienes proporcionan los espacios naturales de la comunidad de Choccocconiri e ir dando valor a lo que es un área representativa y debido a la ubicación tiene un clima especial de los nueve tipos climáticos bien diferenciados en Puno, con todos aspectos geomorfológicos e la comunidad se da una valoración alta de sus recursos geológicos, por lo que debería ser una propuesta de geoparque. 


\section{Agradecimientos}

Se agradece al Centro de Recursos en Geociencia, en especial al director Orlando Poma por la conducción en la elaboración del presente artículo de investigación, también de manera especial a la comunidad de Choccocconiri resaltando su hospitalidad y apoyo en los días de trabajo en campo, también la traducción y guía de la Señora Lourdes Elias.

\section{Referencias}

Acosta, J., Rivera, M., Valencia, H., Chirif, D., \& Huanacuni, I. (2010). Potencial Minero del Perú, Producciòn y Recursos de Oro, Plata y Cobre. Metalogenéticas del Perú.

Almeida, L. (1997). egetación, fitogeografía y paleoecología del zacatonal alpino y bosques montanos de la región central de México. Universidad de Amsterdam, Tesis, 450.

Betejtin, A. (1975). Curso de Mineralogía. MIR Moscú, 739pp.

Calle, M. (1991). Incursión de un Frente Frío en la Selva Peruana y su Efecto en los Cultivos. Tesis de Ingeniero Meteorólogo UNALM, Lima Perú, 384.

Castillo, N., Inche, H., \& Rivera, A. (2011). Geología Preliminar de las Propiedades Mineras de Corporación Minera Ananea. UNAC Puno, 15.

Chávez, A. (2011). Prospección Geoquímica de Sedimentos de Quebradas y Rocas de Zonas de Anomalías Espectrales. EPIG. FIGIM. UNAP. Perú.

Chavez, A., Salas, G., \& Cuadros, J. (1996). Geología de Rinconada. Boletín UNSA Arequipa, 66.

David, B. (1992). Diccionario Geológico. 2da edicion. Instituto Geológico Minero y Metalúrgico Lima-Perú, 1006 pág.

Evaluación de riesgos. (2007). En FAO, Instrumentos de la FAO sobre biodeguridad. Roma.

Flores, A. (2003). evolucion de sus relieves y modelados. universidad nacional de Colombia, 312.

G Hamilton, J. T. (1978). Aspects of a Polar Outbreak leading to frost. Climate, 135pp.

Garreaud, R. (1998). Summertime Incursión of Midlatitude Air Into Subtropical and Tropical South America. Bulletin of the American Meteorological Society, 126.

Goldschmidt, V. (1937). Clasificación Geoquímica de los Elementos de las Fases Principales que Componen la Tierra. Chem. Soc., 673.

Hartman, H. (1987). Estudio Geoquimico de prospección. New Yersey, 234.

Huang, W. (1991). Petrología. Uteha Mexico, 564.

INTIA. (09 de Junio de 2017). Meteorología y climatología . Obtenido de Meteorología y climatología : www.intiasa.es

Jeroenvrp. (09 de Junio de 2017). Climas Brasil. Obtenido de Clima Brasil: http://www.unique-southamerica-travel-experience.com/clima-de-brasil.html

Kappelle, K. (1996). Los bosques de robles (Quercus) en la cordillera de Talamanca, Costa Rica. iNBio, Universidad de Amesterdam, 319p.

Köppen, W. (09 de Junio de 2017). Meteorología y climatología . Obtenido de Clasificación climática de Köppen : http://meteo.navarra.es/definiciones/koppen.cfm

Laubacher, G. (1978). Estudio Geológico de la Región Norte del Lago titicaca. IGM Boletin 5, 120 pp. 
M Seluchi, C. C. (1999.). Intercambios de masas de aire entre latitudes tropicales y extra tropicales de Sudamérica: Validación del modelo regional ETA. Climanálise., , 4.

Marengo, J. (1884). Estudio Sinóptico Climático de los Friajes (Friajens) en la Amazonía Peruana. Revista forestal del Perú, 80pp.

Palacios, M. (2002). Estudio geologico detallado del la región Sur Oeste del Perú. INGEMET, 23.

Palacios, M. (2002). Geológico detallado de la Región Sur Oeste del Perú. INGEMET PERU.

Petersen, M. (1980). Factores de Eriquesimiento de Algunos Elementos, de yacimientos peruanos. Universidad Católica, $27 \mathrm{pp}$.

Pulgar. (1941). regiones naturales del Perú. ercera Asamblea General del Instituto Panamericano de Geografía e Historia.

R Barry, G. C. (1972). Atmósfera Tiempo y Clima. Ediciones Omega, sa. Barcelona, 395 pp.

Rivera, M. (2011). Geología General edicion 2. Editorial Megabyte., 419pp.

Rose, A. (1979). Alteración hidrotermal . Nueva York, 1173-235.

Senamhi. (09 de Junio de 2017). Servicio Nacional de Meteorologóa e Hidrología del Perú. Obtenido de Perú Clima: http://www.peruclima.pe/?p=mapa-climatico-delperu

Sillitoe, R. H. (2010). Alteraciones Hidrotermales y Independent Consultat. Mineralium Deposita, 787- 812.

Tumialan, A. (2003). Manual Geología de Perú. EPIG. FIGIM. UNAP - Puno.

weatherbase. (09 de Junio de 2017). weatherbase. Obtenido de weatherbase Climate: http://www.weatherbase.com/weather/weather-

summary.php3? $=604511 \&$ cityname $=\mathrm{Puno} \% 2 \mathrm{C}+\mathrm{Puno} \% 2 \mathrm{C}+\mathrm{Peru} \& u n i t s=$ 